\title{
Parametri di qualità delle acque imbottigliate: confronto del contenuto degli elementi naturali
}

\author{
Alessia Santoro $^{1}$ \\ ${ }^{1}$ Affiliation not available
}

\begin{abstract}
L'acqua è l'elemento fondamentale per la salute dell'uomo, e proprio per questo motivo esso deve essere in grado di recepire sin da subito informazioni sulla tipologia di acqua da consumare in base alle proprie esigenze tramite una lettura consapevole dell'etichetta.

Un'importante questione da affrontare è la distinzione tra le acque potabili per il consumo umano e le acque minerali, andando a considerare in entrambe i casi quali sono gli elementi mineralogici in esse contenuti e quali sono i valori standard secondo la normativa nazionale.

Lo scopo finale di tale indagine è quello di sottolineare come lo sviluppo delle normative abbia permesso al consumatore una scelta più consapevole dell'utilizzo delle acque minerali imbottigliate.
\end{abstract}

\section{Acque potabili ed acque minerali naturali}

La tematica acqua è diventata e diventa, con il passare del tempo, sempre di maggiore importanza ed attualità, sia per quanto riguarda l'importanza di questa risorsa, sia per una serie di interessi socio-economici a livello globale ${ }^{1}$.Proprio su quest' ultimo fronte sappiamo che il nostro paese detiene il record mondiale nella produzione e nel consumo di acqua minerale naturale e, contemporaneamente, risulta essere anche ai vertici europei per il consumo pro-capite di acqua potabile. 
La Federazione italiana delle industrie delle acque minerali naturali e delle acque di sorgente (Mineracqua), per esempio, con una certa soddisfazione dice che il volume di acqua minerale venduta negli ultimi 15 anni è cresciuto di 3 miliardi di litri, arrivando a 14,8 miliardi nel $2018^{2}$.

Innanzitutto bisogna fare chiarezza sulla differenza tra acque potabili ed acque minerali naturali.

Con la definizione "Acque potabili" o "Acque destinate al consumo umano", si intendono le acque distribuite tramite pubblici acquedotti, ma anche in cisterne, in bottiglie e altri contenitori rispondendo ai requisiti indicati dal D. Lgs. 2 febbraio 2001 n. 31. Inoltre le acque destinate al consumo umano devono essere salubri e pulite, ovvero non devono contenere microrganismi e parassiti, né altre sostanze, in quantità o concentrazioni tali da rappresentare un potenziale pericolo per la salute umana.

Per "Acque minerali naturali", si intendono le acque che, avendo origine da una falda o giacimento sotterraneo, provengono da una o più sorgenti naturali o perforate e che hanno caratteristiche igieniche particolari e, eventualmente, proprietà favorevoli alla salute così come riportato nell' Art. 2 del D. Lgs. 8 ottobre 2011 n. 176 sulla base del D.M. 29 dicembre 2003 n. 301.

Tali Decreti legislativi, come possiamo vedere in Tabella 1, mettono in evidenza come siano differenti per le due "tipologie" di acqua i parametri che li definiscono.

Le acque minerali si distinguono dalle acque potabili per la purezza originaria, il tenore di minerali, oligoelementi o altri costituenti ed, eventualmente, per taluni loro effetti positivi sulla salute, inoltre non vengono sottoposte a nessun procedimento di potabilizzazione e qualsiasi trattamento di disinfezione.

Le acque minerali naturali provengono per lo più da acque meteoriche che filtrano attraverso vari strati del terreno, fino a costituire una falda acquifera. Con questo processo le acque si depurano in parte delle scorie acquisite durante il ciclo atmosfera-suolo e si arricchiscono di sostanze minerali e gassose del terreno, in relazione alle condizioni di pressione e temperatura locali e alla solubilità 
delle rocce con cui vengono a contatto. Così, le caratteristiche chimico-fisiche riflettono in gran parte il paesaggio litogeologico che attraversano durante il loro ciclo idrogeologico ${ }^{3}$.

Avremo, quindi, acque prevalentemente ricche di un elemento minerale o di un altro a seconda della tipologia della roccia dominante nel bacino di pertinenza, della solubilità delle componenti mineralogiche e del tempo di contatto tra acque di circolazione sotterranea e substrato poroso.

Per ogni elemento viene data una definizione descrittiva di uno stato elevato, buono, moderato, scarso e cattivo. Ciascuna autorità nazionale dovrebbe stabilire standard per gli elementi più rilevanti per le pressioni affrontate dal corpo idrico sotto la propria responsabilità e classificare le acque di conseguenza. Lo stato chimico è classificato solo in due categorie: "buono" e "non buono" 4.

\section{Due "tipologie" a confronto}

Dopo aver affrontato la questione acqua potabile ed acqua minerale, comprendiamo come, per tutta una serie di motivi, i due prodotti possono presentare caratteristiche molto diverse, di cui il consumatore deve essere informato.

Basti pensare all'acqua minerale imbottigliata (Fig.2): sulla variazione degli elementi naturali nelle acque minerali è stato effettuato un studio su 371 acque minerali italiane imbottigliate, utilizzando la composizione chimico-fisica e chimica riportata sulle loro etichette, secondo i limiti standard stabiliti dalle principali normative italiane e internazionali per i parametri selezionati. Sono state prese in considerazione anche le normative e le linee guida più rappresentative nel mondo per confrontare la qualità delle acque imbottigliate italiane, tra cui la Direttiva 2003/40/CE della Comunità Europea (UE), il Real Decreto 140/2003 del 7 febbraio per la Spagna e i regolamenti nazionali 
sull'acqua potabile primaria e secondaria (USEPA, 2006) sviluppato dalla Environmental Protection Agency degli Stati Uniti d'America. Cinquantadue sono i parametri chimico-fisici e chimici considerati,tra i più importati: $\mathrm{Al}, \mathrm{As}, \mathrm{B}, \mathrm{Ba}, \mathrm{Br}, \mathrm{Ca}, \mathrm{Cl}, \mathrm{CO} 2, \mathrm{Cr}, \mathrm{Cu}, \mathrm{Fe}$, durezza, $\mathrm{Mg}, \mathrm{Mn}, \mathrm{Na}$, $\mathrm{Pb}, \mathrm{pH}, \mathrm{Zn}{ }^{5}$.

Dovrebbe essere la cosa più naturale del mondo scegliere un' acqua destinata al consumo giornaliero. Ma quali elementi analizzare? Sapore, gradevolezza o effetti biologici? Va subito evidenziato il fatto che non esiste un'acqua minerale migliore di tutte le altre; ogni consumatore può trovare tra quelle presenti sul mercato quella più adatta alle sue esigenze. E, quindi, può essere di grande aiuto la conoscenza delle caratteristiche di un'acqua minerale per orientare le scelte del consumatore. A questo scopo una mano ci viene data dalla serie di elementi obbligatori che devono essere riportati in etichetta tra cui, in particolare, l'indicazione della composizione analitica con i componenti caratteristici. Spesso, sono proprio questi ultimi che ci permettono di scegliere non solo l'acqua più adatta ai nostri gusti ma talvolta quella che presenta le caratteristiche dieto-terapeutiche più appropriate alla nostra situazione.

Ma l'acqua in bottiglia può sempre essere considerata "sicura"? Basti pensare che molte persone usano bottiglie d'acqua per lunghi periodi di tempo e le conservano a temperatura ambiente o in condizioni di calore estremo all'aperto, una potenziale preoccupazione è che potrebbe esserci una crescita batterica nell' acqua in bottiglia che potrebbe avere potenziali implicazioni per la salute. Pertanto, è importante indagare se c'è una crescita batterica nel tempo nell' acqua in bottiglia, una volta aperta e utilizzata, e studiare gli effetti della temperatura sulla qualità dell'acqua in bottiglia per quanto riguarda la contaminazione microbica ${ }^{6}$.

Nonostante in Italia siano attualmente presenti sul territorio un numero sempre crescente di marche di acque minerali, non bisogna denigrare l'utilizzo di acqua potabile, in quanto sono in crescita anche nuove tecnologie e metodi per la depurazione dell'acqua: uno di questi metodi è la raccolta e il trattamento dell'acqua piovana che fornisce acqua direttamente alle famiglie consentendo ai 
membri della famiglia di avere il pieno controllo del proprio sistema idrico, il che riduce notevolmente i costi di funzionamento e manutenzione degli impianti centralizzati ${ }^{7}$. Gli impianti per la depurazione dell'acqua però devono essere tenuti sotto controllo in quanto la ricerca scientifica sul controllo della qualità delle acque e la presenza di virus nelle acque potabili e, in generale, nel processo di trattamento e riutilizzo delle acque reflue ha prodotto numerose evidenze di come tali patogeni possono entrare in contatto con l'uomo e risultare la causa di un cospicuo numero di malattie in assenza di impianti di trattamento ${ }^{8}$. Un altro metodo molto valido per la depurazione dell'acqua è il processo UV-basato di ossidazione avanzata (UV/AOP) utilizzato per la disinfezione e la rimozione dei contaminanti ${ }^{9}$.

Sono diversi i motivi che possono spingere il consumatore a preferire la cosiddetta acqua del rubinetto (Fig.3) a quella imbottigliata, e viceversa, ma è facile intuire come l'acqua minerale imbottigliata abbia un notevole impatto ambientale. Di seguito viene riportata la Tabella 2 in cui vengono evidenziati pro e contro di acqua del rubinetto ed acqua minerale.

Per valutare e confrontare l'impatto sull'ambiente e sulle risorse dell'acqua del rubinetto e dell'acqua minerale naturale imbottigliata , è stata definita una metodologia Footprint, che integra Ecological Footprint (EF), Water Footprint (WF) e Carbon Footprint (CF). La metodologia si ispira al Life Cycle Thinking, che prescrive di valutare il carico ambientale di un prodotto considerando ogni fase del suo intero ciclo di vita. Al fine di ottenere informazioni su tali passaggi e su qualsiasi processo a monte e a valle coinvolto, è stata eseguita una valutazione del ciclo di vita di ciascun modo di bere ${ }^{10}$.

Nel caso di mancanza di acque da poter imbottigliare, come accade in altri paesi del mondo, possiamo ben capire come e acque sotterranee sono un'importante risorsa idrica per scopi potabili" e che "al giorno d'oggi, gli studi sulle risorse idriche sotterranee evidenziano molti fattori che influenzano la qualità e la variabilità della chimica delle acque sotterranee come le attività antropiche, il 
deflusso, le condizioni di drenaggio, la litologia, il rifornimento e il clima" ${ }^{11}$, fattori che risultano essere diversi da quelli della normativa italiana in quanto le relative tecnologie di purificazione dell' acqua risultano di più complessa attuazione.

\section{Conclusione}

L'Italia è la nazione con la più alta produzione e consumo di acque minerali al mondo, nonostante i metodi di purificazione delle acque siano bene sviluppati per permetterci di poter usufruire anche di una buona acqua potabile. Grazie alla normative vigenti e agli studi scientifici il consumatore viene maggiormente tutelato, in quanto può essere capace di scegliere l'acqua più adatta alle sue esigenze, sia per quanto riguarda il contenuto degli elementi minerali in essa presente, sia per quanto riguarda l'aspetto dell'impatto che questa ha.

I risultati di questo studio mettono in evidenza come una lettura consapevole delle etichette delle acque ci permetta di effettuare una scelta ragionata in base alla composizione di minerali che può risultare utile, non solo per individuare quella più adatta a una particolare situazione patologica, ma anche per riequilibrare situazioni fisiologiche temporaneamente alterate: ad esempio le acque leggere poco mineralizzate, con $\mathrm{pH}$ leggermente acido, favoriscono lo smaltimento di scorie metaboliche; In gravidanza, è utile raccomandare l'assunzione di acqua (in genere 2 1/die) classificata a "media mineralizzazione" e "calcica" dato l'intenso utilizzo del calcio durante questo periodo; Lo sportivo, al termine dell'attività fisica, trae vantaggio dal consumo di un'acqua mineralizzata "contente bicarbonato" e con un residuo fisso di circa $1 \mathrm{~g} / \mathrm{l}$ sia per reintegrare la perdita di liquidi e di sali dovuta alla sudorazione sia per favorire l'eliminazione delle scorie azotate e correggere l'acidosi determinata dalla fatica muscolare. 


\section{References}

1.Temporelli, G. \& Mantelli, F. Acque potabili e minerali naturali: le nuove disposizioni di legge in riferimento ai parametri chimici. Rivista dell'associazione idrotecnica italiana L'Acqua 4, (2004).

2.Pira, R. L. Acqua minerale: i consumi italiani sono da record mondiale. Un disastro ambientale ed economico, ma l'industria è soddisfatta - Il Fatto Alimentare. (2019).

3.Mosca, M., Sanzini, E. \& Aureli, P. Le acque minerali naturali: come non perdersi in un bicchiere d'acqua'.. Notiziario dell'Istituto Superiore di Sanita 19.10 11-14, (2006).

4.Naddeo, V., Zarra, T. \& Belgiorno, V. Optimization of sampling frequency for river water quality assessment according to Italian implementation of the EU Water Framework Directive. Environmental Science \& Policy 10, 243-249 (2007).

5.Naddeo, V., Zarra, T. \& Belgiorno, V. A comparative approach to the variation of natural elements in Italian bottled waters according to the national and international standard limits. Journal of Food Composition and Analysis 21, 505-514 (2008).

6.Raj, S. D. Bottled Water: How Safe Is It?. Water Environment Research 77, 3013-3018 (2005).

7.Naddeo, V., Scannapieco, D. \& Belgiorno, V. Enhanced drinking water supply through harvested rainwater treatment. Journal of Hydrology 498, 287-291 (2013).

8.Naddeo, V. Il coronavirus nelle acque. doi:10.22541/au.158263992.20053407.

9.Feola, R. Riutilizzo delle acque: cosa sono le clorammine e qual è il loro ruolo nei trattamenti avanzati?. doi:10.22541/au.157919031.14329406.

10.Botto, S. Tap Water vs. Bottled Water in a Footprint Integrated Approach. Nature Precedings (2009) doi:10.1038/npre.2009.3407.1.

11.Baghdadi, M. E., Zantar, I., Jouider, A., Nadem, S. \& Medah, R. Evaluation of hydrogeochemi- 
cal quality parameters of groundwater under urban activities-Case of Beni Mellal city (Morocco). Euro-Mediterranean Journal for Environmental Integration 4, (2019). 


\section{Figure Captions}

Figure 1. Acqua (unsplash)

Figure 2. Differenze legislative delle due tipologie di acqua (ARPA - Snpambiente)

Figure 3. Acqua minerale imbottigliata (unsplash)

Figure 4. Acqua potabile proveniente direttamente dai nostri rubinetti (unsplash)

Figure 5. Pro e contro di acqua del rubinetto ed acqua minerale ( Culligan.it) 
Figures

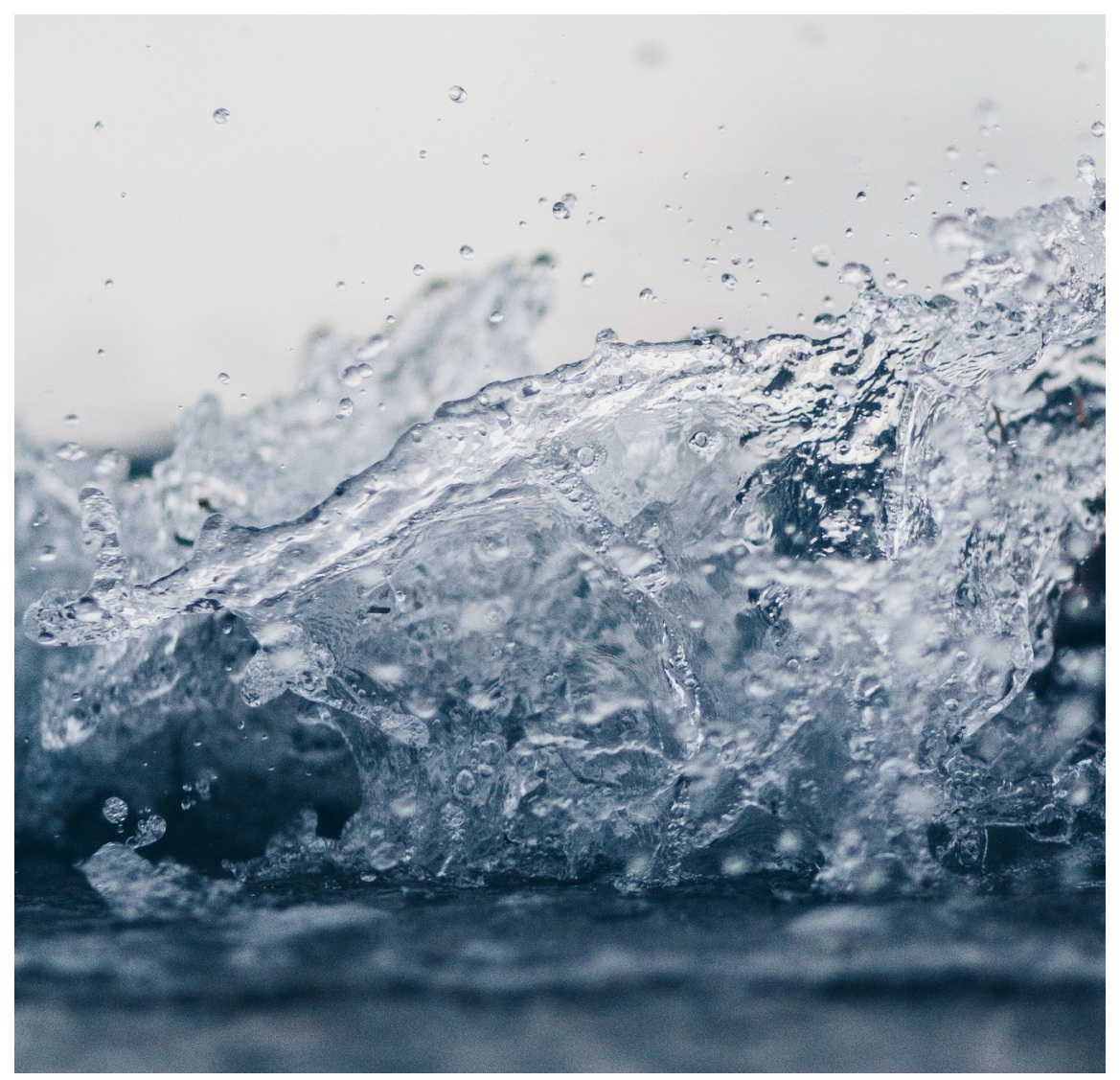

Figure 1: Acqua (unsplash) 


\begin{tabular}{|c|c|c|}
\hline & $\begin{array}{c}\text { ACQUE DESTINATE AL CONSUMO } \\
\text { UMANO }\end{array}$ & ACQUE MINERALI NATURALI \\
\hline PARAMETRO & $\begin{array}{c}\text { D. Lgs. } 2 \text { febbraio } 2001 n^{\circ} 31 \\
\text { Attuazione della direttiva 98/83/CE }\end{array}$ & $\begin{array}{c}\text { D.M. } 29 \text { dicembre } 2003 \\
\text { Attuazione della direttiva } 2003 / 40 / C E\end{array}$ \\
\hline Torbidità & $\begin{array}{l}\text { Accettabile per i consumatori e senza } \\
\text { variazioni anomale }\end{array}$ & Parametro non previsto \\
\hline Odore & $\begin{array}{c}\text { Accettabile per i consumatori e } \\
\text { senza variazioni anomale }\end{array}$ & Parametro non previsto \\
\hline Sapore & $\begin{array}{l}\text { Accettabile per i consumatori e senza } \\
\text { variazioni anomale }\end{array}$ & Parametro non previsto \\
\hline Colore & $\begin{array}{c}\text { Accettabile per i consumatori e } \\
\text { senza variazioni anomale }\end{array}$ & Parametro non previsto \\
\hline Temperatura & Parametro non previsto & Parametro previsto ma senza limite \\
\hline $\mathrm{pH}$ & $\begin{array}{c}6,5 \div 9,5 \\
\text { L'acqua non deve essere aggressiva } \\
\text { Per le acque frizzanti confezionate in } \\
\text { bottiglie o contenitori il valore può } \\
\text { scendere sino a } 4,5\end{array}$ & Parametro previsto ma senza limite \\
\hline Conducibilità $(\mu \mathrm{S} / \mathrm{cm})$ a $20^{\circ} \mathrm{C}$ & 2500 & Parametro previsto ma senza limite \\
\hline Cloruri & $\begin{array}{c}250 \mathrm{mg} / \mathrm{l} \\
\text { L'acqua non deve essere aggressiva }\end{array}$ & Parametro previsto ma senza limite \\
\hline Solfati & $250 \mathrm{mg} / 1$ & Parametro previsto ma senza limite \\
\hline Silice & Parametro non previsto & Parametro previsto ma senza limite \\
\hline Calcio & Parametro non previsto & Parametro previsto ma senza limite \\
\hline Magnesio & Parametro non previsto & Parametro previsto ma senza limite \\
\hline Sodio & $200 \mathrm{mg} / \mathrm{l}$ & Parametro previsto ma senza limite \\
\hline Potassio & Parametro non previsto & Parametro previsto ma senza limite \\
\hline Alluminio & $200 \mu \mathrm{g} / 1$ & Parametro previsto ma senza limite \\
\hline Residuo fisso $\left(180^{\circ} \mathrm{C}\right)$ & $1500 \mathrm{mg} / \mathrm{l}$ (valore massimo consigliato) & Parametro previsto ma senza limite \\
\hline Anidride carbonica & Parametro non previsto & Parametro previsto ma senza limite \\
\hline Bicarbonati & Parametro non previsto & Parametro previsto ma senza limite \\
\hline Ferro & $200 \mu \mathrm{g} / 1$ & Parametro previsto ma senza limite \\
\hline Manganese & $50 \mu \mathrm{g} / 1$ & $500 \mu \mathrm{g} / \mathrm{l}$ conformità entro il $31 / 12 / 2004$ \\
\hline Rame & $1,0 \mathrm{mg} / \mathrm{l}$ & $1,0 \mathrm{mg} / \mathrm{l}$ \\
\hline Fosforo & Parametro non previsto & Parametro previsto ma senza limite \\
\hline Fluoro & $1,50 \mathrm{mg} / \mathrm{l}$ & $\begin{array}{c}5,0 \mathrm{mg} / \mathrm{l} \\
\text { Segnalazione in etichetta oltre } 1,5 \mathrm{mg} / \mathrm{l} \text { (acque } \\
\text { destinate all'infanzia) } \\
\text { conformità entro il 31/12/2006 }\end{array}$ \\
\hline Mercurio & $1,0 \mu \mathrm{g} / 1$ & $1,0 \mu \mathrm{g} / 1$ \\
\hline Nichel & $20 \mu \mathrm{g} / \mathrm{l}$ & $\begin{array}{c}20 \mu \mathrm{g} / \mathrm{l} \\
\text { conformità entro il 31/12/2006 }\end{array}$ \\
\hline Piombo & $\begin{array}{c}10 \mu \mathrm{g} / 1 \\
\text { Deroga sino al 31/12/2013(25 } \mathrm{gg} / \mathrm{l})\end{array}$ & $10 \mu \mathrm{g} / 1$ \\
\hline
\end{tabular}

Figure 2: Differenze legislative delle due tipologie di acqua (ARPA - Snpambiente) 


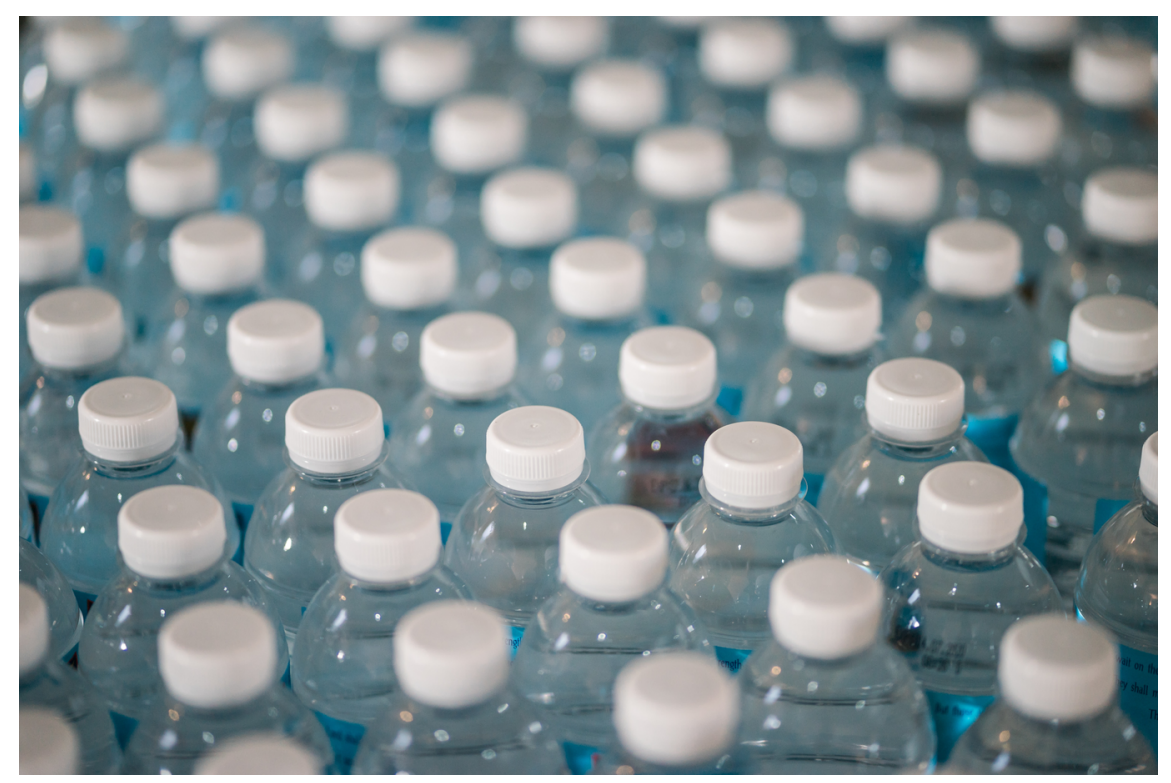

Figure 3: Acqua minerale imbottigliata (unsplash)

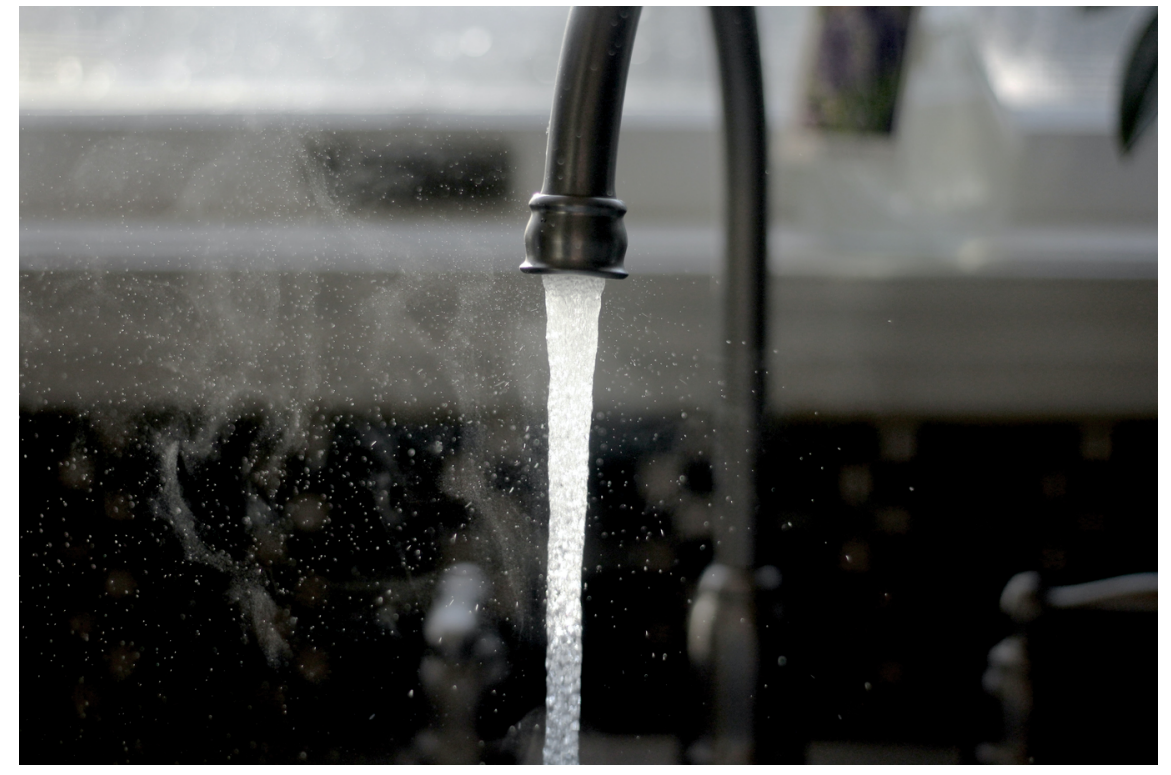

Figure 4: Acqua potabile proveniente direttamente dai nostri rubinetti (unsplash) 


\begin{tabular}{|c|c|c|}
\hline & ACQUA DEL RUBINETTO & ACQUA MINERALE \\
\hline Origine & Qualsiasi & Sotterranea \\
\hline Trattamenti consentiti & $\begin{array}{l}\text { Tutti quelli necessari per la conformità al } \\
\text { D.Lgs } 31 / 2001\end{array}$ & $\begin{array}{l}\text { Tutti quelli che non ne modificano le } \\
\text { caratteristiche e le proprietà }\end{array}$ \\
\hline Disinfezione & Necessaria & Vietata \\
\hline Trasporto & Rete di distribuzione & Bottiglie \\
\hline Conservazione & Nessuno & Anche 2 anni \\
\hline $\begin{array}{l}\text { Possibili cause di } \\
\text { alterazione }\end{array}$ & $\begin{array}{l}\text { Rilascio di sostanze metalliche dalle } \\
\text { tubazioni e possibile proliferazione batterica } \\
\text { in presenza di serbatoi non puliti e depositi } \\
\text { calcarei }\end{array}$ & $\begin{array}{l}\text { La plastica (PET) può rilasciare sostanze } \\
\text { tossiche come l'acetaldeide e la formaldeide }\end{array}$ \\
\hline Costo in Euro/Litro & $0,0018 € /$ litro $(* 1)$ & $0,21 € /$ litro $(* 2)$ \\
\hline Impatto ambientale & Basso & $\begin{array}{c}\text { Molto elevato, dovuto alla } \\
\text { produzione/trasporto/smaltimento dei } \\
\text { contenitori in plastica }\end{array}$ \\
\hline
\end{tabular}

Figure 5: Pro e contro di acqua del rubinetto ed acqua minerale ( Culligan.it) 